\title{
Odontogenic cutaneous fistula of the face
}

\author{
Keiichi Ohta DDS, Hitoshi Yoshimura DDS PhD
}

Cite as: CMAJ 2019 November 18;191:E1281. doi: 10.1503/cmaj.190674

A $\mathrm{n}$ otherwise healthy 61-year-old woman was referred to us by her dermatologist with a 6-month history of an enlarging nodule of the left cheek. The patient was aware of slight mobility of the left posterior maxillary teeth. She had no alcohol or drug problems and was not homeless. Her oral health knowledge and attitude were good. Extraoral examination showed an erythematous, crusted nodule measuring about $50 \mathrm{~mm}$ with pus discharge on the left cheek (Figure 1A). Intraoral examination showed swelling of the left posterior maxillary buccal gingiva and hypermobility of the left maxillary teeth without pain (Figure 1B). Computed tomography showed radiolucency in the periapex and perforation of the buccal cortical bone of the left maxillary teeth with surrounding soft-tissue edema that was consistent with the site of the cutaneous nodule (Appendix 1, available at www.cmaj. ca/lookup/suppl/doi:10.1503/cmaj.190674/-/DC1). Blood examination did not show any major abnormalities. Odontogenic cutaneous fistula was diagnosed. Cefdinir was administered for 12 days, and the patient underwent extraction of the infected teeth. Nine days later, the nodule was resolved, with no complications.

Odontogenic cutaneous fistula is a rare extraoral path of chronic dental infection caused by an untreated tooth..$^{1-3}$ It is more common among middle-aged and older adults and women. ${ }^{3}$ Most cases occur as a nodule in the mandibular angle or chin. ${ }^{3}$ Only about $50 \%$ of patients present with a history of toothache. ${ }^{1}$ The lesion is often misdiagnosed as being of nonodontogenic origin, which results in inappropriate treatment, such as a prolonged course of antibiotics and repeated surgical treatment. ${ }^{1}$ Identifying a heavily decayed, discoloured or mobile tooth leads to suspicion of odontogenic origin. Radiologic examination shows a radiolucent lesion in the periapex of the affected tooth. ${ }^{1}$ The differential diagnosis includes pyogenic granuloma, tuberculous infection, salivary gland fistula, suppurative lymphadenitis and carcinoma. ${ }^{1,2,4}$ Treatment of the infected tooth leads to resolution within 2 weeks. ${ }^{1}$

\section{References}

1. Sammut S, Malden N, Lopes V. Facial cutaneous sinuses of dental origin - a diagnostic challenge. Br Dent J 2013;215:555-8.

2. Kishore Kumar RV, Devireddy SK, Gali RS, et al. Cutaneous sinuses of cervicofacial region: a clinical study of 200 cases. J Maxillofac Oral Surg 2012;11:411-5.

3. Guevara-Gutiérrez E, Riera-Leal L, Gómez-Martínez M, et al. Odontogenic cutaneous fistulas: clinical and epidemiologic characteristics of 75 cases. Int J Dermatol 2015;54:50-5.

4. Lee EY, Kang JY, Kim KW, et al. Clinical characteristics of odontogenic cutaneous fistulas. Ann Dermatol 2016;28:417-21.

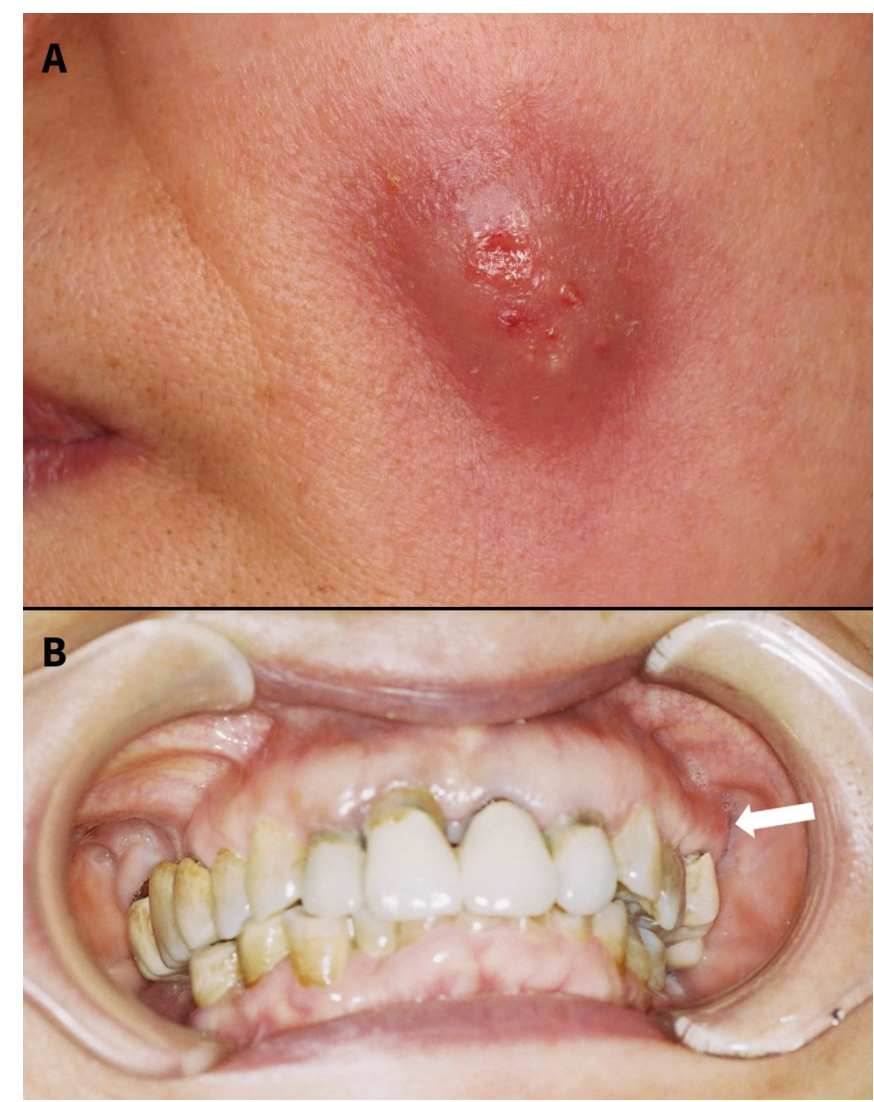

Figure 1: (A) Erythematous, crusted cutaneous nodule measuring about $50 \mathrm{~mm}$ on the left cheek of a 61-year-old woman with an odontogenic cutaneous fistula. (B) Swelling and redness of the left posterior maxillary buccal gingiva (arrow).

\section{Competing interests: None declared.}

This article has been peer reviewed.

The authors have obtained patient consent.

Affiliation: Department of Dentistry and Oral Surgery, Unit of Sensory and Locomotor Medicine, Division of Medicine, Faculty of Medical Sciences, University of Fukui, Fukui, Japan

Correspondence to: Keiichi Ohta, kohta@g.u-fukui.ac.jp 